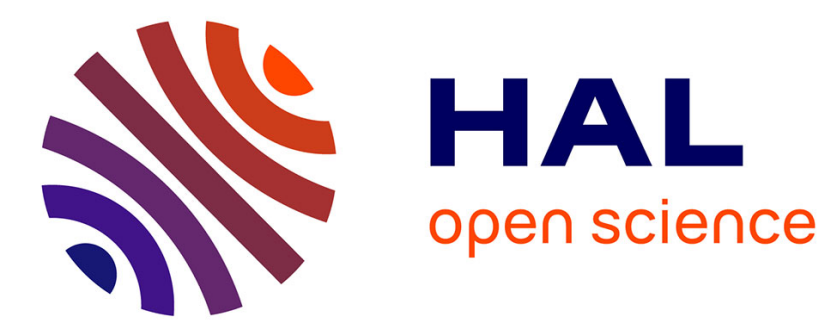

\title{
L'usage de la poésie haïku en psycho-oncologie
}

Alfonso Santarpia, M. Paul, E. Dudoit

\section{To cite this version:}

Alfonso Santarpia, M. Paul, E. Dudoit. L'usage de la poésie haiku en psycho-oncologie. Revue Francophone de Psycho-Oncologie, 2015, 9 (2), pp.127-134. 10.1007/s11839-015-0523-5 . hal-01164410

\section{HAL Id: hal-01164410 \\ https://hal-amu.archives-ouvertes.fr/hal-01164410}

Submitted on 16 Jun 2015

HAL is a multi-disciplinary open access archive for the deposit and dissemination of scientific research documents, whether they are published or not. The documents may come from teaching and research institutions in France or abroad, or from public or private research centers.
L'archive ouverte pluridisciplinaire HAL, est destinée au dépôt et à la diffusion de documents scientifiques de niveau recherche, publiés ou non, émanant des établissements d'enseignement et de recherche français ou étrangers, des laboratoires publics ou privés. 
Santarpia, A., Paul, M., \& Dudoit, E. (2015). L'usage de la poésie haïku en psycho-oncologie. Psycho-Oncologie, 9 (2), 127-134.

\section{L’usage de la poésie haïku en psycho-oncologie}

The use of Haiku poetry in psycho-oncology

Santarpia Alfonso 1, Paul Melanie 1, Dudoit Eric 2,

1 Aix Marseille Université, LPCLS EA 3278, 13621, Aix en Provence, France.

2 Service d'oncologie médicale, AP-HM, Timone, F-13385 Marseille, France.

Conflit d'intérêt : aucun.

*Auteur Contact (premier auteur) : Alfonso SANTARPIA

Maître de Conférences, Aix-Marseille Université (AMU), Aix-en-Provence, France.

Laboratoire de Psychopathologie Clinique: langage et subjectivité (LPCLS) Email_asantarpia@yahoo.it Tel. 00330689896635 


\section{Résumé}

Objectif : Cette étude qualitative et exploratoire vise à décrire les effets d'un protocole poétique centré sur les haïkus en psycho-oncologie chez un patient atteint d'un cancer.

Matériel et Méthodes : Il s'agit d'un protocole d'écriture poétique composé de 4 étapes: un entretien préliminaire, quinze haïkus (sans le vers du milieu) tirés de grands auteurs japonais, réalisation d'un poème composé librement par deux patients atteints d'un cancer, un entretien final sur l'expérience de l'écriture poétique. Cas clinique : un homme, Mr D âgé de 25 ans qui est en traitement pour un cancer. Résultats: Nous montrons les variations discursives utilisées avant et après l'expérience de l'écriture poétique, à travers le logiciel Tropes V8.4. Conclusion : Le travail poétique avec la poésie Haïku peut être un outil de travail utile, dans un contexte de support clinique en institution ou comme préparation à un travail de psychothérapie. Nous pensons que la structure formelle des haïkus puissent inviter à un travail poétique spécifique composé de : évocation poétique, synthèse et schématisation des vécus plus intimes en milieu oncologique.

Mots clés: Poésie Haïku, Poésie Thérapie, analyse du discours, cancer, psycho-oncologie, logiciel Tropes V8.4.

\section{$\underline{\text { Abstract }}$}

Aims: Our study aims to describe the discourse effects of specific and structured protocol focused on several Haiku poems about one man, who have experienced cancer. Procedure: the protocol consists of 4 steps; a preliminary exploratory interview, fifteen Haiku proposed without a part of poem (a creative writing, filled in by the patient), a free form haiku composed by the patient and finally a final interview. Clinical Case: One man, Mr D aged 25, He had tumor. Results: Using this protocol, we showed discourse variations on the illness before and after the experience of poetry writing, by Tropes V8.4 software. Conclusion: Haiku poetry can be a useful tool in the context of supportive interventions or as preparatory work for engagement in psychotherapeutic intervention. We believe that the formal structure of haikus can create conditions for a specific poetic work composed of: poetic evocation, synthesis and mapping of the most intimate experiences in oncology environment.

Keywords: Poetry Haïku, Poetry Therapy, cancer, psycho-oncology, discourse analysis, Software Tropes V8.4. 


\section{Introduction}

« Le poème s'obtient par la coagulation de mots semblables aux gouttes d'un sang épais et noir : sur le linge blanc de page, le sang qui s'égoutte est celui d'une blessure. Amas de cellules cancéreuses, placenta sanglant, les mots étouffent le langage, l'engorgent... ».

Pierre-Jean Jouve, Sueur de Sang, 1933-1935.[1]

Affronter le cancer de nos jours n'est pas seulement une question de médecine technoscientifique de traitements médicaux, mais aussi affronter une maladie qui évoque des représentations sociales [2] de type métaphorique ce qui influence les vécus et les signifiants engagés dans le «travail de la maladie » [3-5]. Menzel [6] fut un des premier à illustrer les représentations métaphoriques [7] du cancer comme «une lutte » ou encore « une guerre entre les cellules maladie et l'organisme ». Stolberg [8] souligne l'usage massif en occident de la métaphore du cancer comme « une armée de cellules hostiles qui attaquent le corps et doivent être repoussées » ou comme « un killer silencieux » [9]. Susan Sontag [10], romancière américaine, en 1976 à l'âge de 43 ans, subit une mastectomie et se rétablit progressivement d'un cancer du sein, elle écrit pendant de longs mois sur sa maladie. Elle dénonce dans son essai aussi vif qu'argumenté l'abus de langage figuré social qui ferait de la maladie cancéreuse une métaphore. Son but, dégager le cancer de toutes les fausses images, les mythologies et les métaphores qui lui sont attachées : le «Crabe », une maladie qui consume le corps, l'idée que certains tempéraments psychologiques y sont plus exposés que d'autres. Comme si ce type de métaphore évoquant des représentations sociales pouvait empêcher un face à face authentique avec la maladie et ses métaphores individualisées, plus proches à la singularité du patient. En fait, les vécus des patients peuvent prendre la forme de métaphores créatives ${ }^{1}$ ou d'images du langage plus ou moins

\footnotetext{
${ }^{1}$ Dans cet article, nous allons définir la métaphore et la métaphorisation comme toute phrase, énoncé renvoyant à : a) une similarité et/o une association entre deux catégories d'organisation expérientielle différente (sensation, perception, abstraction); b) un processus d'imagerie et de simulation créative dans un état de conscience ordinaire ou modifié ; c) un effet de mobilisation de significations nouvelles pour le
} 
complexes comme l'exprime une patiente (après une ablation totale des seins) qui se comparait à « une extra-terrestre » [13] :

« Je me mets en maillot je me mets une prothèse, je ne sors jamais sans ma prothèse. C'est moi. Au contraire. C'est moi qui...qui regarde tout le monde et qui dis ouais tiens elle c'est une femme et moi je ne suis la...je suis plus une femme hè...je...je suis peut-être une extra...enfin dans ma tête je suis une extra-terrestre quoi. Il me manque quelque chose. Ma féminité a morflé c'est sûr et certain ...une femme est faite avec deux seins, deux bras, deux jambes...donc là il me manque quelque chose...il me manque quelque chose ».

Autre témoignage de ce langage figuré [14] avec le récit d'une autre patiente:

« On m'a levé le cœur quand on m'a levé le sein, ils ont tout pris..., je suis sale, pourrie de l'intérieur, je voudrais frotter, frotter à la Javel pour enlever cette crasse, sortir mes organes et les remettre une fois propres, laver ce sang noir, c'est possible vous pensez... et non je le sais bien... Je voudrais m'envoler comme un oiseau, être libre, je serai un corbeau... ».

Le récit convoque des images d'un «corps sale», d'un «sang noir », d'un « corps contenant » dans lequel on pourrait entrer et sortir. Un travail psychique ciblé sur une reconfiguration des vécus angoissants du cancer à travers de nouvelles métaphores et des images créatives pourrait apporter de nouveaux horizons thérapeutiques pour les patients en psycho-oncologie. Le domaine de la poésie, lieu privilégié du langage figuré, se prête bien à ce type de reconfiguration. Actuellement la Poésie-Thérapie (Poetry Therapy), est un domaine d'intervention thérapeutique qui présente une littérature abondante [15-18] avec une revue internationale scientifique : The Journal of Poetry Therapy. Selon les auteurs, la poésie aurait le pouvoir d'aider les patients dans les changements de vie consécutifs aux maladies graves. Des références à ce modèle de thérapie sont trouvés autant dans la littérature psychiatrique [19-21] et que dans celle consacrée aux psychothérapies [22]. cet article s'inspire de travaux de Cameron et de Ritchie $[11,12]$ 
Ainsi, Mazza [23] et Reiter [24] ont identifié les processus thérapeutiques caractéristiques de la Poesie-Therapie:

- Développer sa créativité (avoir de nouvelles idées, éveiller l'insight et rechercher des informations ainsi augmenter l'estime de soi) ;

- Favoriser le travail de symbolisation à travers l'usage de métaphores et de contes ;

- Améliorer la capacité de communiquer et de s'exprimer à travers une expression langagière plus figurée.

- Produire une redécouverte sensorielle à travers l'écriture.

D'autres recherches ont identifié les processus en jeu : retrouver des objets psychiques perdus dans la mémoire [25], favoriser l'imagination [26] et un sens esthétique d'harmonie et de symétrie [26], schématiser et synthétiser l'imagination productrice [27].

Ces processus thérapeutiques sont typiques des thérapies humanistes [28, 29] mais aussi de nouvelles approches en psychologie cognitive [30]. Bacqué [31] a proposé une réflexion sur le travail poétique en psycho-oncologie : «La poésie est composée de métaphores linguistiques élaborées, même si parfois elles prennent pour point de départ des images très crues. Ce qui frappe, c'est la capacité de franchir plusieurs registres, de partir de la fange ou de miasmes... pour arriver à l'élévation la plus transcendantale ». Nous orientons cette recherche sur l'hypothèse qu'il pourrait être utile en psycho-oncologie de développer cet outil qu'est le travail poétique pour favoriser la mobilisation psychique telle que l'évoque Bacqué, et qui peut même aboutir à «l' élévation la plus transcendantale », comme l' argumente la littérature d'orientation humaniste sur la Poésie-Thérapie [11-16]. Celle-ci postule un besoin fondateur de création et d'esthétique [28, 29]. Le travail poétique va plus loin que la seule expression émotionnelle ou la catharsis. En particulier, nous nous attachons à comprendre le possible usage d'un type spécifique de poésie « le haïku » comme modalité d'élaboration créative de la souffrance psychique en oncologie. Ce type de psychothérapie a été déjà utilisé dans la clinique de la schizophrénie [30] pour sa capacité à préciser le vécu de ces patients. Le Haïku est aussi utilisé comme support pédagogique en 
neurosciences[32]. Le Haïku [33] est donc un petit poème (d'origine japonaise), s'écrivant en trois lignes : court - long - court (classiquement 5-7-5 syllabes). Il comporte un mot qui le relie à la réalité et, en général, à la nature. Dans les Haïkus « libres », cette référence peut être absente. Il comporte une césure, qui fait basculer le texte d'une image dans une autre. Etant très bref, le haïku permet de saisir des choses très petites, des moments presque invisibles. Le Haïku offre l'aspect d'un condensé poétique, assez suggestif pour orienter la rêverie, assez flou pour la libérer [34, 35].

\section{Objectif de la recherche}

Il s'agit d'une étude clinique de type qualitatif, selon le modèle inductif qui part du cas singulier ou de plusieurs cas singuliers pour en tirer des observations potentiellement généralisables. A partir du travail poétique d'un patient atteint de cancer, nous allons décrire et analyser les processus psychiques mobilisés dans ses écrits poétiques sous forme de haïkus. L'analyse va consister à suivre les variations discursives du patient sur le récit de sa maladie, avant et après celle-ci, au travers de son travail d'écriture poétique sous forme d'haïkus mais également au cours des 2 entretiens proposés avant et après le travail d'écriture. Notre hypothèse est que ce type de travail créatif peut influencer non seulement le discours sur le vécu du cancer mais aussi diminuer la souffrance psychique qui lui est associée.

\section{Méthodologie}

\section{Présentation des patients et consentement}

Cette recherche a été menée par une stagiaire en psychologie. Un patient a accepté de participer à l'étude. Mr D âgé de 25 ans, atteint d'un Sarcome d'Ewing (en traitement) vivant en couple, sans enfant, Web designer de profession. La stagiaire lui a proposé d'effectuer un travail autour de l'écriture poétique qu'il a donc accepté. Le patient a signé un consentement de participation dans lequel il est écrit que le patient peut sortir de l'étude à tout moment, et que les données le concernant sont strictement confidentielles. Au niveau de l'équipe médico-soignante et du psychologue référent du service d'oncologie, le projet 
d'un atelier d'écriture poétique a été accueilli avec curiosité et intérêt et a fait l'objet d'une présentation dans laquelle la stagiaire psychologue a pu illustrer la complexité des différentes stratégies d'écriture (voir paragraphe matériel et procédure). Les ateliers d'écriture ne sont pas encore très répandus ni connus dans les services de cancérologie. Tous les entretiens ont été enregistrés à l'aide d'un dictaphone pour retranscrire de façon précise les entretiens dans l'après-coup. Le style de présence de la stagiaire dans la rencontre avec les patients s'inscrit dans l'approche humaniste de Carl Rogers [36] : support empathique, acceptation, écoute et participation active dans la dynamique intersubjective.

\section{Matériel et Procédure}

Nous avons mis au point un protocole d'écriture poétique selon la technique SADUPA [37] ciblé sur quatre phases organisé sur 4-6 semaines selon les conditions du patient:

1) Un entretien préliminaire dans lequel différents thèmes existentiels sont abordés : Corps, Maladie, Famille et Créativité. Il a été effectué dans une modalité semi-directive à travers l'usage de trois types de relances, l'interrogation, la déclaration et la réitération [35]. Il s'agit d'entrer en contact avec les patients et de collecter leurs discours sur les vécus de la maladie et la disponibilité à faire un travail relativement engageant de type créatif.

2) Proposition de 15 haïkus (voir annexe A), tirés de grands auteurs japonais ; 3 poèmes pour chacun de ces thématiques existentielles, « La mort », «L'amour », « La spiritualité », « Le temps », « La sensorialité ». La stagiaire psychologue les lit tous aux patients. Dans un deuxième temps, elle présente les haïkus qu'elle a lus sans le vers du milieu. La consigne qui est demandée aux patients est de compléter (exemples, annexe B) les haïkus afin de leur donner un sens personnel. La stagiaire psychologue relit le travail pour faire du lien avec les patients.

3) Dans cette phase les patients doivent présenter un poème composé de plusieurs haïkus ; il l'a réalisé seul chez lui ou à l'hôpital. Il les lit devant la stagiaire psychologue. 
l'interrogation, la déclaration et la réitération [35]. Elle porte sur le Corps, la Maladie, la Famille et la

Créativité après l'expérience poétique.

\section{Le travail d'écriture poétique}

Dans le tableau 1, la colonne centrale correspond au travail d'apprentissage poétique du patient. Il doit compléter et écrire en poésie le vers manquant.

\begin{tabular}{|c}
\hline Ligne 1 \\
\hline Lire dans le lac \\
\hline Me voici \\
\hline Aubépine en fleur \\
\hline Cheminant par la vaste lande \\
\hline On vieillit \\
\hline Dans les jeunes herbes \\
\hline Tout en ce monde \\
\hline Les papillons bat les ailes \\
\hline Nos belles années \\
\hline Matin de printemps \\
\hline Pour ce regard là \\
\hline Rien qui m'appartienne \\
\hline Le temps qui ne s'efface rien \\
\hline Une carpe saute \\
\hline Dans tes bras j'oublie \\
\hline
\end{tabular}

Patient Monsieur D
toujours à l'affut
machine que je suis
regardant le passé
marchant à reculons
parce que la vie
fraicheur du matin
devient magique
suivant le vent
à nous contempler
soleil jaunissant
je ferais n'importe quoi
seulement mon corps
filant à toute vitesse
à la vue de la barque
les malheurs que j'ai subis

\begin{tabular}{c}
\hline Ligne 3 \\
\hline Ces rides sur l'eau \\
\hline Est sans limite \\
\hline Et moi qui vieillis \\
\hline Pèsent sur moi \\
\hline Est source de larme \\
\hline Oublie ses racines \\
\hline A contempler les fleurs \\
\hline De ce monde \\
\hline Sous le soleil de Juillet \\
\hline Déborde de vie \\
\hline Dans tous les feux de l'enfer \\
\hline Et la fraicheur de l'air \\
\hline Pour les amours forts \\
\hline A la lune d'automne \\
\hline Amour amnésie
\end{tabular}

Tableau 1. Les énoncés (en italique) de Monsieur D qui a complété la structure des haïkus «à vers manquant ».

Suite au travail d'apprentissage technique le patient peut écrire un Haïku libre.

Haïku de Monsieur D (phase 3, écriture originale, annexe C)

Je ne suis plus/le pessimiste/d'autre fois

Il fait beau/L'été arrivé /Sortons un peu

Je mange des fruits/Ça m'enchante/C'est vrai

Je suis tombé par terre /En glissant/Sur une pierre

\section{Analyse sémantique du corpus: Tropes V8.4}

Nous avons réalisé une analyse sémantique à l'aide du logiciel Tropes (VF8.4, www.tropes.fr) pour l'entretien préliminaire et final afin d'avoir une idée des catégories de discours utilisées par le patient. L'utilisation de ce type de logiciel permet d'avoir une analyse quantitative d'un texte (transcription d'entretien, prise de notes, écriture libre, etc.) 
en le découpant en différentes catégories selon le lexique employé par les sujets (verbes, adjectifs, substantifs, etc.). Il permet également la création d'un dictionnaire (Scénario) personnalisé par le chercheur et ajouté sur le corpus discursif spécifique.

\section{Résultats}

Nous allons montrer d'abord les substantifs (ex. maladie, cancer), les adjectifs, les verbes, les pronoms dans les entretiens préliminaires et dans les entretiens finaux qui illustrent les vécus après le travail d'écriture poétique du patient.

\section{Le dictionnaire de Monsieur D}

Tropes 8.4 nous a donné les caractéristiques quantitatives du corpus: 353 substantifs avec une moyenne de 1 occurrence pour chaque catégorie de substantif, 199 verbes avec une moyenne de 7 occurrences pour chaque catégorie de verbes, 93 adjectifs avec une moyenne de 3 occurrences pour chaque catégorie de adjectifs. Pour réaliser l'analyse du contenu concernant la thématique de la maladie, on a sélectionné à travers le logiciel Tropes 8.4 les termes ${ }^{2}$-cibles avec les occurrences suivants : (Cancer, 11), (Corps, 6), (Cortisone, 3), (Déni, 2), (Fruit, 5), (Malade, 8), (Maladie, 15), (Mort, 3), (Pierre, 4), (Sport, 5), (Traitement, 5), (Tumeur, 1), (Vie, 10), (Accepter, 8), (Grandir, 4), (Guérir, 4), (Intéresser, 2), (Manger, 19), (Mourir, 3), (Reculer, 2), (Relativiser, 4), (Ressentir, 4), (Rêver, 1), (Sentir, 2), (Sortir, 16), (Sourire, 4), (Vivre, 3), (Beau, 7), (Content, 7), (Cool, 7) (Difficile, 16), (Frais, 3), (Horrible, 3), (Impossible, 2), (Mal, 3), (Pessimiste, 2), (Plein, 1), (Poétique, 1), (Serein, 1), (Seul, 6), (Triste, 3), (Vif, 1), (Vivant, 1).

\section{Comparaison Catégories de mots entre l'entretien préliminaire et l'entretien final}

Nous allons présenter les graphiques de comparaison de mots cibles dans l'entretien préliminaire et dans l'entretien final.

\footnotetext{
2 Les verbes sont ramenés à l'infinitif, les noms au singulier et les adjectifs au masculin singulier (lemmatisation) dans les tableaux. Tropes 8.4 calcule les occurrences des pluriels des noms, des adjectifs et les conjugaisons et les formes des verbes.
} 


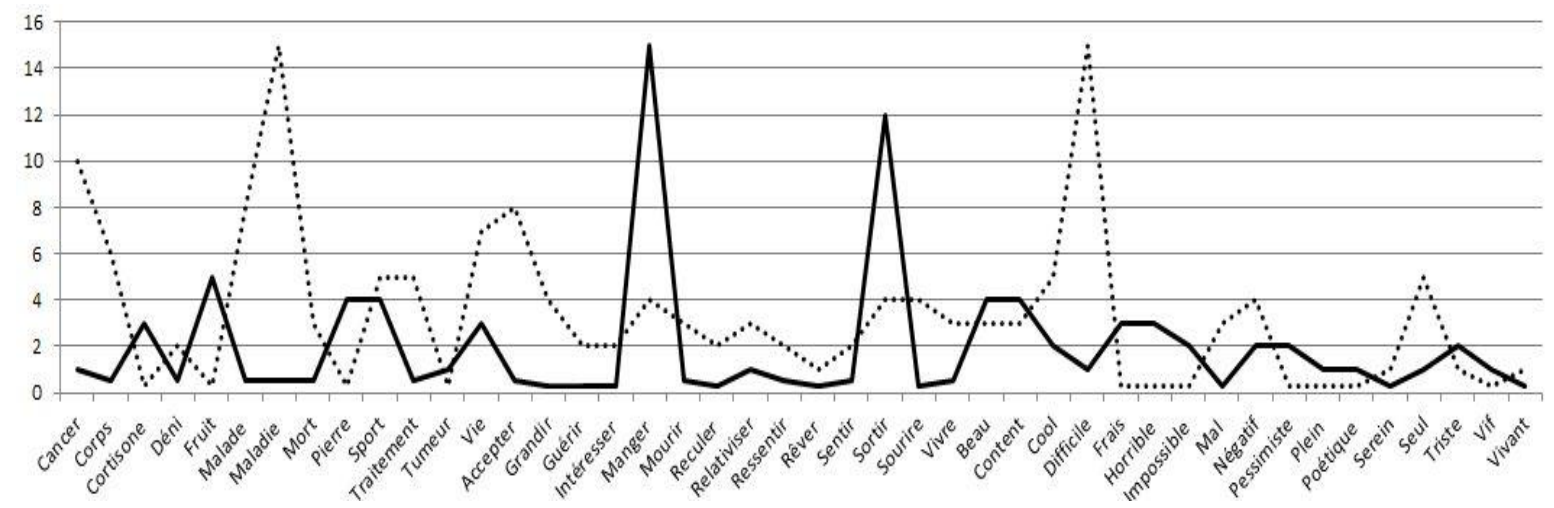

\section{METTRE LE GRAPHIQUE 1 (pièce jointe)}

Graphique 1. En ligne en pointillé les mots-cibles de l'entretien préliminaire, en noir foncé les mots-cibles de l'entretien final de Monsieur D.

\section{Le récit de la maladie dans l'entretien préliminaire ${ }^{3}$ :}

\section{Discours de Monsieur D :}

\section{Le temps du déni de la maladie}

«C'est du déni. Je n'ai pas accepté ça car j'ai été jamais malade de ma vie, j'ai jamais eu un petit «p» de travers et le fait d'apprendre ça, ben je ne voulais pas. Je ne voulais pas car c'est à l'encontre de soi. C'est pas tu ne veux pas parce que voilà mais c'est :j'y peux rien, l'inconscient et au final en parlant, oui c'est du déni, que je ne voulais pas l'admettre maintenant j'y suis. C'est-à-dire que quand on l'apprend, c'est difficile parce qu'on a du mal à l'accepter parce que l'on assimile toujours le cancer avec la mort. Donc du coup on se dit, enfin moi je me suis dit : j'ai 25 ans comment je vais faire. Je vais mourir, comment ça va se passer etc ».

\section{La perte de cheveux comme temps d'acceptation de la maladie}

Donc quand je dis on, je parle pour moi mais je parle aussi pour ma maman et ma petite sœur qui l'ont pris très mal forcément. C'est plus ou moins normal. Moi ça a eu un effet à retardement. C'est-à-dire que quand le professeur m'a appris que j'avais ce cancer-là, j'ai eu du mal à l'accepter. Enfin je l'ai accepté plus ou moins mais je me suis voilé la face et c'est quand j'ai commencé à perdre mes cheveux en fait, mes dreadlocks que je portais depuis 10 ans. Quand j'ai commencé à les perdre, fin décembre 2013 là je me suis dit bon ben mon coco tu rentres dans la maladie.

\section{Les possibilités inattendues d'être malade}

Dans un sens moi, je suis bien content, enfin c'est bizarre ce que je vais dire mais je suis bien content d'être tombé malade. Parce que ça me fait grandir et je vais en ressortir deux fois voire trois plus fort qu'auparavant et que je pourrais boucler la bouche de certaines personnes.

\section{L'optimisme}

Pour ma part je préfère les choses enjouées, jolies, belles plutôt que les choses négatives qui ne nous font pas avancer et qui nous font plutôt reculer.

\section{La force de la solitude}

Donc j'ai trouvé des moyens pour battre la nausée, pour battre les effets secondaires qui font que du coup j'ai besoin d'être seul. Pour la partie traitement je dirais qu'être seul c'est pas mal car ça permet de te retrouver et d'acquérir quand même une certaine force qui te permet d'avancer.

\footnotetext{
${ }^{3}$ En noir foncé les mots-cibles identifiées sur le logiciel Tropes 8.4.
} 


\section{$\underline{\text { Le récit de la maladie dans l'entretien final }}$}

\section{La vitalité inspirée par le beau temps de l'été}

Après le deuxième Haïku : il fait beau, l'été arrive, sortons un peu. C'est parce qu'il fait beau je me languis de sortir. Parce qu'être alité, rester à la maison 24h/24, j'en peux plus. Donc je croise les doigts qu'avec les dernières chimio.

\section{L'éveil sensoriel ciblé sur le gout}

Bon le troisième haïku c'est : je mange des fruits, ça m'enchante, c'est frais. (il rit) Ben c'est parce que je bouffe énormément de fruits en ce moment. Genre là j'ai mangé du melon d'hiver à midi, j'étais trop content c'était très bon: oui je mangeais une pomme. D'ailleurs j'en mange tous les matins. Je mange beaucoup de fruits depuis que je suis avec ma copine.

\section{La chute sur la pierre comme possibilité de se relever}

Après mon haïku c'est :je suis tombé par terre en glissant sur une pierre. Je ne sais pas je l'ai marqué comme cela. Je ne suis tombé pas vraiment. ouais ouais c'est dans l'ordre, je les ai écrits à la suite. :ouais je passe du coq à l'âne. Je sais pas je peux marquer n'importe quoi en fait., non bien sûr que non. Je suis un peu tout blanc, un peu tout noir. Je change d'humeur très rapidement. Ouais mais pour ma part ce n'est pas forcément négatif aussi. Parce que le fait de tomber et ben moi ça me permet de me relever. Je me suis relevé toujours. Donc ce n'est pas forcément négatif. On peut l'interpréter comme cela. Oui voilà tu glisses sur une pierre, ça va. T'es tombé pas tout seul ça c'est sûr aussi. Il y a cette chute, mais elle a été causée. Ce n'est pas toi qui as décidé de chuter, oui j'ai glissé.

\section{Le recours à l' Haïku comme mise en sens dans une temporalité instantanée}

Et vu que c'était frais du coup, j'ai trouvé ça rigolo, j'ai regardé sur internet quelques haïkus. Et puis je me suis dis allez je vais en faire un petit. J'ai mis même pas trois minutes pour en faire. Ben ouais: tu vois ils ne sont pas tous très poétiques. Mais bon j'aime bien en fait. Je pourrais en faire toute la journée. Tu t'installes et quand, tu as une idée derrière la tête, tu l'écris et c'est fini. C'est que 3 vers donc ça va vite.

\section{Discussion}

Ces résultats décrivent les variations discursives des récits de la maladie d'un patient atteint de cancer avant et après un travail d'écriture poétique centré sur les haïkus. Ces résultats confirment ce qui a été décrit dans la littérature [12-16-24] sur les effets de la PoésieThérapie, à savoir un changement de discours qui témoigne une mise au travail psychique sur l'expérience du cancer. Chez Monsieur D, on observe au niveau quantitatif et dans l'entretien final, qu'il ne fait plus référence comme lors de l'entretien préliminaire, aux termes tels que «maladie », «malade», «mort ». De même, il n'apparaît plus dans son discours de processus défensif classique [36] comme le déni du cancer (tous ces termes ont 0 occurrences, dans l'entretien final, voir graphique 1), et le discours se centre beaucoup sur le goût (manger, occurrences 15). 
Au niveau plus qualitatif, nous pouvons souligner l'effet expressif/sensoriel [23] du travail poétique, par exemple dans la phrase : «Bon le troisième haïku c'est : je mange des fruits, ça m'enchante, c'est frais. Ben c'est parce que je bouffe énormément de fruits en ce moment. Genre là j'ai mangé du melon d'hiver à midi, j'étais trop content c'était très bon: oui je mangeais une pomme », ou l'effet de symbolisation à travers la métaphore [23] de la chute sur la pierre de Monsieur D : «Parce que le fait de tomber et ben moi ça me permet de me relever. Je me suis relevé toujours. Donc ce n'est pas forcément négatif. On peut l'interpréter comme cela. ». Il y a également un passage discursif en faveur de la structure brève et spécifique de l'Haïku [23] qui supporte la pertinence de notre choix de recherche : «j'ai trouvé ça rigolo, j'ai regardé sur internet quelques haïkus. Et puis je me suis dit, allez je vais en faire un petit. J'ai mis même pas trois minutes pour en faire...Tu t'installes et quand, tu as une idée derrière la tête, tu l'écris et c'est fini. C'est que 3 vers donc ça va vite ». Un travail d'effet de prise de conscience est également noté « Je ne sais pas je peux marquer n'importe quoi en fait, non bien sûr que non. Je suis un peu tout blanc, un peu tout noir. Je change d'humeur très rapidement ».

\section{Conclusion}

A partir du travail poétique effectué par un patient atteint de cancer, nous avons cherché à montrer comment cet outil thérapeutique peut influencer le discours sur la maladie et le vécu de l'expérience du cancer. Le Haïku fondé sur un type de structures poétiques brèves a permis la production d'un discours plus élargi et moins chargé d'émotions négatives.

Cette étude préliminaire a pour objectif de développer ce type d'atelier d'écriture en cancérologie et de le proposer à un plus grand nombre de participants afin d'approfondir et de mieux évaluer l'impact thérapeutique de ce type de psychothérapie en individuel ou en 
groupe chez les patients atteints de cancer. La richesse thématique et le pouvoir évocateur des haïkus invitent le patient à se penser différemment et à penser différemment aussi, son rapport à la maladie. La poésie, plus spécifiquement la poésie Haïku, peut constituer en psychologie clinique (en particulier en psycho-oncologie) une ressource psychothérapeutique précieuse pour accompagner «le travail de la maladie» [3] des patients atteints de cancer et peut-être même, les aider à parvenir à «l'élévation la plus transcendantale »[31].

\section{Remerciements :}

À Havsteen-Franklin Dominik (Consultant in Arts Psychotherapies, CNWL NHS Foundation Trust Roundwood Resource Centre, London), pour les attentives suggestions sur la forme en anglais de l'article.

On remercie particulièrement Sylvie Pucheu, membre du comité éditorial de Psycho-oncologie et psychologue à l'HEGP qui a effectué un travail précieux de correction et de questionnement.

Déclaration de conflit d'intérêt : L'auteur déclare ne pas avoir de conflit d'intérêt.

\begin{tabular}{|c|c|c|c|}
\hline $\begin{array}{c}\text { Cheminant par la vaste lande } \\
\text { Les hauts nuages } \\
\text { Pèsent sur moi. } \\
\text { Yosa Buson }\end{array}$ & $\begin{array}{c}\text { Dans les jeunes herbes } \\
\text { Le vieux saule } \\
\text { Oublie ses racines. } \\
\text { Yosa Buson } \\
\end{array}$ & $\begin{array}{c}\text { Rien qui m'appartienne } \\
\text { Sinon la paix du cœeur } \\
\text { Et la fraîcheur de l'air. } \\
\text { Kobayashi Issa } \\
\end{array}$ & $\begin{array}{l}\text { Le papillon bat des ailes } \\
\text { Comme s'il désespérait } \\
\text { De ce monde. } \\
\text { Kobayashi Issa }\end{array}$ \\
\hline $\begin{array}{c}\text { Matin de printemps - } \\
\text { Mon ombre aussi } \\
\text { Déborde de vie! } \\
\text { Kobayashi Issa } \\
\end{array}$ & $\begin{array}{c}\text { Tous en ce monde } \\
\text { Sur la crête d'un enfer } \\
\text { À contempler les fleurs! } \\
\text { Kobayashi Issa } \\
\end{array}$ & $\begin{array}{c}\text { On vieillit - } \\
\text { Même la longueur du jour } \\
\text { Est source de larmes. } \\
\text { Kobayashi Issa } \\
\end{array}$ & $\begin{array}{c}\text { Me voici } \\
\text { Là où le bleu de la mer } \\
\text { Est sans limite. } \\
\text { Taneda Santoka } \\
\end{array}$ \\
\hline $\begin{array}{c}\text { Une carpe saute - } \\
\text { Des rides } \\
\text { À la lune d'automne. } \\
\text { Masaoka Shiki } \\
\end{array}$ & $\begin{array}{c}\text { Nos belles années } \\
\text { Sur la plage abandonnées } \\
\text { Soleil de juillet } \\
\text { Kirikino } \\
\end{array}$ & $\begin{array}{c}\text { Aubépine en fleur } \\
\text { À l'aube d'un jour nouveau. } \\
\text { Et moi qui vieillis... } \\
\text { Kirikino } \\
\end{array}$ & $\begin{array}{c}\text { Lire dans le lac } \\
\text { Reflets de réalités } \\
\text { Ces rides sur l'eau } \\
\text { Kirikino } \\
\end{array}$ \\
\hline $\begin{array}{c}\text { Le temps qui n'efface rien } \\
\text { Un accroche-cœur } \\
\text { Pour les amours fortes } \\
\text { Yosa Buson } \\
\end{array}$ & $\begin{array}{c}\text { Pour ce regard là } \\
\text { J'ai jeté mon cœur } \\
\text { Dans tous les feux de l'enfer } \\
\text { Djabal-Al-Tariq }\end{array}$ & $\begin{array}{c}\text { Dans tes bras j'oublie } \\
\text { Que le monde fout le camp } \\
\text { Amour amnésie } \\
\text { Yosa Buson }\end{array}$ & Haïkus \\
\hline
\end{tabular}

Annexe A - 15 Haïkus proposés [37, 38]
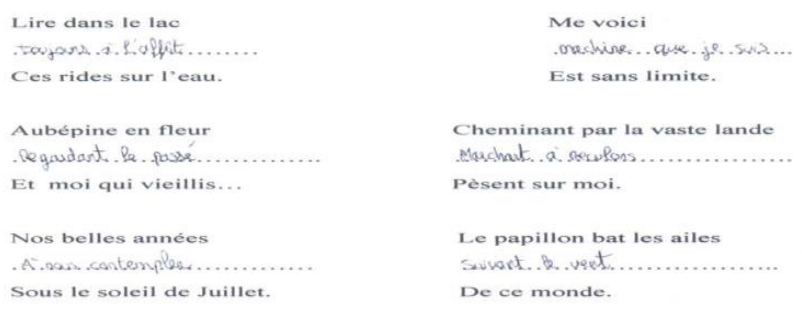
Annexe B - Exemple de Monsieur D qui complète le vers manquant.

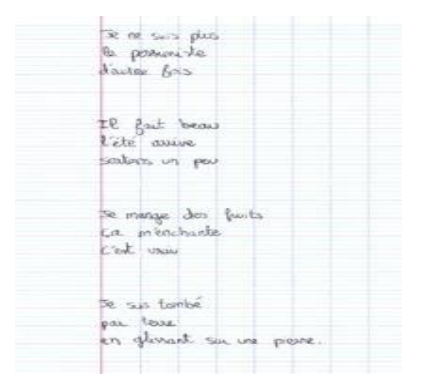

Annexe C - Haïku libre de Mr D

\section{Références}

1. Jouve PJ (1966) Sueur de Sang. Collection de Poche Galimard, Paris

2. Durkheim E (1898) Représentations individuelles et représentations collectives. Rev Métaphys Morale VI:272-302.

3. Pedinielli J-L (1993) Psychopathologie du somatique : La "maladie-du-malade" [Somatic psychopathology: The "illness-of-the-ill"]. Clin Méditerranéennes 37/38:121-137.

4. Pedinielli J-L (1986) Le "travail de la maladie" chez les insuffisants respiratoires chroniques. Thèse de doctorat dirigé par Beauchesne Hervé, Université Paris 5.

5. Pedinielli J-L (1999) Les théories personnelles des patients [Patients' personal theories]. Prat Psychol 4:53-62.

6. Menzel R (1954) Contre le cancer, Editions Pierre Horay. Paris

7. Murphy GL (1996) On metaphoric representation. Cognition 60:173-204.

8. Stolberg M (2014) Metaphors and Images of Cancer in Early Modern Europe. Bull Hist Med 88:48-74.

9. Jasen P (2009) From the "silent killer" to the "whispering disease". Ovarian cancer and the uses of metaphor. Med Hist 53:489-512.

10. Sontag S, Paloméra M-F de (1979) La maladie comme métaphore. Christian Bourgois Editeur, Paris

11. Cameron L (1999) Identifying and describing metaphor in spoken discourse data. Res. Appl. Metaphor

12. Ritchie LD (2009) Relevance and Simulation in Metaphor. Metaphor Symb 24:249262. doi: 10.1080/10926480903310310

13. Santarpia A, Tellène J, Carrier M (2013) Les effets d'un protocole d'écriture poétique SANTEL sur l'image érotique du corps dans le traitement du cancer féminin : étude pilote. Psycho-Oncol 7:156-162. doi: 10.1007/s11839-013-0426-2

14. Cannone P, Marie D, Dudoit E (2008) Cancer du sein avancé. Springer Paris, Paris

15. Blanton S (1960) The healing power of poetry. Thomas Crowell, New York 
16. Leedy J (1969) Poetry therapy: The use of poetry in the treatment of emotional disorders. Lippincott, Philadelphia

17. Prescott F (1922) The poetic mind. New York: Macmillan. Macmillan, New York

18. Shrodes C (1949) Bibliotherapy. A theoretical and clinical experimental study. University of California

19. Shelton D (1999) Healing words. Washington, DC: AMA Staff News. http://amaassn. org/scipubs/amnews/pick 99/feat0517.htm. Accessed 25 Apr 2003

20. Langosch D (1987) The use of poetry therapy with emotionally disturbed children. Am J Soc Psychiatry 7:97-100.

21. Houlding S, Holland P (1988) Contributions of a poetry writing group to the treatment of severely disturbed psychiatric inpatients. Clin Soc Work J 16:194-200.

22. McLoughlin D (2000) Transition, transformation, and the art of losing: Some uses of poetry in hospice care for the terminally ill. Psychodyn Couns 6:215-234.

23. Mazza M (1999) Poetry therapy: Interface of the arts and psychology. CRC Press, Boca Raton

24. Reiter S (1997) Poetry therapy: Testimony on Capitol Hill. J Poet Ther 10:169-178.

25. Clancier A (2001) Le travail du poème.: Figurabilité et poésie. Rev Fr Psychanal 65:1283. doi: $10.3917 / \mathrm{rfp} .654 .1283$

26. Gergen K (2000) Die poetische Dimension: Therapeutische Moglichkeiten. In: Deissler K, McNamee S (eds) Phil Sophie Auf Couch Soz. Poesie Ther. Gesprche. Heidelberg: Carl-Auer-Systeme Verlag., pp 97-108

27. Ricoeur P (1982) «Imagination et métaphore ». Psychol. Medicale 14:

28. Maslow A (1943) A theory of human motivation. Psychol Rev 50:370-396.

29. Maslow A (1972) Vers une psychologie de l’Être. Fayards, Paris

30. Collins KS, Furman R, Langer CL (2006) Poetry therapy as a tool of cognitively based practice. Arts Psychother 33:180-187. doi: 10.1016/j.aip.2005.11.002

31. Bacqué M-F (2013) Le «travail de la maladie» revisité par la psycho-oncologie. Psycho-Oncol 7:137-139. doi: 10.1007/s11839-013-0434-2

32. Pollack AE, Korol DL (2013) The use of haiku to convey complex concepts in neuroscience. J Undergrad Neurosci Educ JUNE Publ FUN Fac Undergrad Neurosci 12:A42-48.

33. haïku jardins chimiques. http://www.atomes-crochus.org/haikus/haiku.php.

34. Morier H (1998) Dictionnaire de poétique et de rhétorique, 5. éd. rev. et augm. Presses universitaires de France, Paris

35. Blyth R (1975) Haiku. Hokuseido, Tokyo 
36. Rogers (2009) Psychothérapie et relations humaines. Esf Editeur, Issy les Moulineaux

37. Santarpia A, Dudoit E, Paul M (2015) The Discursive Effects of the Haiku-based SADUPA Poetry Technique in Palliative Care. J. Poet. Ther.

38. Blanchet A (1991) Dire et faire dire. L'entretien. A.Colin, Paris

39. Reich M (2009) Cancer et image du corps : identité, représentation et symbolique. Inf Psychiatr 85:247-254.

40. Atlan C, Bianu Z (2002) Anthologie Haiku: anthologie du poème court japonais. Gallimard, Paris

41. Titus-Carmel J (2004) 66 Haiku. Verdier, Paris 